\title{
Counter Example: The Algorithm of Determinant of Centrosymmteric Matrix based on Lower Hessenberg Form
}

\author{
Nur Khasanah ${ }^{1}$, Farikhin ${ }^{2}$ \\ ${ }^{1}$ UIN Walisongo Semarang, khasanah.nur@walisongo.ac.id \\ ${ }^{2}$ Universitas Diponegoro,farikhin@undip.ac.id
}

doi: https://doi.org/10.15642/mantik.2020.6.1.20-29

\begin{abstract}
Abstrak: Algoritma dalam perhitungan determinan matriks centrosymetric telah diperoleh sebelumnya. Algoritma tersebut menunjukkan tahapan dalam perhitungan komputasi dari determinan yang efisien pada matriks centrosymmetric yaitu dengan melakukan perhitungan pada blok matriksnya saja. Salah satu blok matriks yamng muncul pada algoritma tersebut adalah matriks Hessenberg bawah. Meskipun, bentuk matriks lainnya juga memungkinkan muncul pada perhitungan determianan matriks centrosymmetric. Oleh sebab itu, artikel ini bertujuan untuk menunjukan kemungkinan kemunculan blok matriks centrosymmetric dan bagaimana algoritma yang telah diperoleh, diterapkan dalam menyelesaikan determinan berbagai jenis matriks centrosymmetric. Beberapa contoh dari blok matriks yang berbeda pada determinanan matriks centrosymmetric diberikan pula. Contoh tersebut sangat bermanfaat dalam pemahaman lanjut saat menggunakan algoritma ini dengan berbagai kasus yang berbeda.
\end{abstract}

Kata kunci: Centrosymmetric; Determinan; Matriks blok; Hessenberg bawah

\begin{abstract}
The algorithm for computing determinant of centrosymmetric matrix has been evaluated before. This algorithm shows the efficient computational determinant process on centrosymmetric matrix by working on block matrix only. One of block matrix at centrosymmetric matrix appearing on this algorithm is lower Hessenberg form. However, the other block matrices may possibly appear as block matrix for centrosymmetric matrix's determinant. Therefore, this study is aimed to show the possible block matrices at centrosymmetric matrix and how the algorithm solve the centrosymmetric matrix's determinant. Some numerical examples for different cases of block matrices on determinant of centrosymmetric matrix are given also. These examples are useful for more understanding for applying the algorithm with different cases.
\end{abstract}

Keywords: Centrosymmetric; Determinant; Block matrices; Lower Hessenberg 


\section{Introduction}

Centrosymmetric matrix is the matrix with a special structure at its entries that is symmetry about its center. Some special properties of centrosymmetric matrix about its special structure are observed before [1-9]. Based on the role of orthogonal matrix and special structure of centrosymmetric matrix, the centrosymmetric matrix can be formed as block matrices. These block matrices can be used to construct an efficient algorithm on determinant. The study at [10-14] shows the computational process for inverse and determinant at special matrix, called centrosymmetric matrix.

Moreover, [15] also working at the analytical process for determinant of centrosymmetric matrix with lower Hessenberg as block matrix. The result of the study is only computing block matrix at computational process for determinant centrosymmetric matrix having a half size of centrosymmetric's size. However, this analytical process only aplicable at one example with lower Hesssenberg at certain block matrix at centrosymmetric matrix.

On the other side, based on its applications, the other block matrices also appear at centrosymmetric matrix. By the roles of centrosymmetric matrix [16], then the evaluation of previous algorithm at some block matrices is needed. The different block matrices that possibly appear are upper Hessenberg, centrosymmetric, lower triangular, upper triangular, diagonal, tridiagonal matrix also appears on its. Based on previous study, the algorithm of determinant centrosymmetric matrix can not be applied at general centrosymmetric matrix which will be evaluated at this paper. This study shows the possible different block matrices appear at this matrix and the different treatment for applying the algorithm of determinant of centrosymmetric matrix.

\section{Preliminaries}

In this section, there are some basic properties of centrosymmetric matrix before further discussion on determinant of centrosymmetric matrix.

Definition $1\left[\mathbf{1 4 , 1 5}\right.$. The $A=\left(a_{i j}\right)_{n \times n} \in R^{n \times n}$ is a centrosymmetric matrix, if

$$
a_{i j}=a_{n-i+1, n-j+1}, 1 \leq i \leq n, 1 \leq j \leq n \text { or equivalently } \boldsymbol{J}_{\boldsymbol{n}} \boldsymbol{A} \boldsymbol{J}_{\boldsymbol{n}}=\boldsymbol{A},
$$

with $\boldsymbol{J}_{\boldsymbol{n}}=\left(\boldsymbol{e}_{\boldsymbol{n}}, \boldsymbol{e}_{\boldsymbol{n}-\mathbf{1}}, \cdots, \boldsymbol{e}_{\mathbf{1}}\right)$ and $\boldsymbol{e}_{\boldsymbol{i}}$ is the unit vector with the $i$-th elements 1 and others 0 .

Furthermore, based on the definition of centrosymmetric matrix, by using orthogonal matrix, the properties of this matrix is written as :

Lemma $2[14,15]$. The $\boldsymbol{A}=\left(a_{i j}\right)_{n \times n} \in R^{n \times n}(n=2 m)$ is centrosymmetric matrix, if and only if $\boldsymbol{A}$ has the form:

$$
A=\left(\begin{array}{ll}
B & J_{m} C J_{m} \\
C & J_{m} B J_{m}
\end{array}\right), \text { and } Q^{T} A Q=\left(\begin{array}{cc}
B-J_{m} C & 0_{m} \\
0_{m} & B+J_{m} C
\end{array}\right)
$$

where $\boldsymbol{B} \in R^{m \times m}, \boldsymbol{C} \in R^{m \times m}$ and $Q=\frac{\sqrt{2}}{2}\left(\begin{array}{cc}I_{m} & I_{m} \\ -J_{m} & J_{m}\end{array}\right)$.

Proof. $Q^{T} A Q=\frac{\sqrt{2}}{2}\left(\begin{array}{cc}I_{m} & -J_{m} \\ I_{m} & J_{m}\end{array}\right)\left(\begin{array}{cc}B & J_{m} C J_{m} \\ C & J_{m} B J_{m}\end{array}\right) \frac{\sqrt{2}}{2}\left(\begin{array}{cc}I_{m} & I_{m} \\ -J_{m} & J_{m}\end{array}\right)=\left(\begin{array}{cc}B-J_{m} C & 0_{m} \\ 0_{m} & B+J_{m} C\end{array}\right) \cdot$

Therefore, the computing determinant block matrix only, $\boldsymbol{B}-\boldsymbol{J}_{\boldsymbol{m}} \boldsymbol{C}$ and $\boldsymbol{B}+\boldsymbol{J}_{\boldsymbol{m}} \boldsymbol{C}$, is same as computing determinant of centrosymmetric matrix. 


\section{Results and Discussion}

\subsection{The Algorithm of Determinant of Centrosymmetric Matrix}

Based on the algorithm of determinant of lower Hessenberg matrix, this algorithm can be used to compute the determinant of centrosymmetric matrix with lower Hessenberg as block matrices. The steps of determinant of centrosymmetric matrix with lower Hessenberg form as block matrices are written as follows $[14,15]$.

a. Construct block centrosymmetric matrix

By the Lemma 2 and based on orthogonal matrix then the centrosymmetric matrix is written as block centrosymmetric matrix $\left(\begin{array}{cc}\boldsymbol{B}-\boldsymbol{J}_{\boldsymbol{m}} \boldsymbol{C} & \mathbf{0}_{m} \\ \mathbf{0}_{m} & \boldsymbol{B}+\boldsymbol{J}_{m} \boldsymbol{C}\end{array}\right)$. It can be seen that centrosymmetric matrix has block matrices $\boldsymbol{B}-\boldsymbol{J}_{\boldsymbol{m}} \boldsymbol{C}$ and $\boldsymbol{B}+\boldsymbol{J}_{\boldsymbol{m}} \boldsymbol{C}$.

b. Construct block centrosymmetric matrix on Hessenberg matrix

By the previous explanation, centrosymmetric has block matrices $\boldsymbol{B}-\boldsymbol{J}_{\boldsymbol{m}} \boldsymbol{C}$ and $\boldsymbol{B}+$ $\boldsymbol{J}_{\boldsymbol{m}} \boldsymbol{C}$. These matrices have special form as lower Hessenberg matrix. Based on the definition of centrosymmetric matrix and lower Hessenberg matrix, then the centrosymmetric matrix with lower Hessenberg as block matrices has the form as follows.

$$
A=\left(\begin{array}{ll}
B & J_{m} C J_{m} \\
C & J_{m} B J_{m}
\end{array}\right)
$$

$=\left(\begin{array}{ccccc|ccccc}b_{11} & b_{12} & & & & & & & c_{m, 2} & c_{m, 1} \\ b_{21} & b_{22} & b_{23} & & & & & c_{m-1,3} & c_{m-1,2} & c_{m-1,1} \\ \vdots & \vdots & \ddots & \ddots & & & . & \ddots & \vdots & \vdots \\ b_{m-1,1} & b_{m-1,2} & \cdots & b_{m-1, m-1} & b_{m-1, m} & c_{2, m} & c_{2, m-1} & \cdots & c_{22} & c_{21} \\ b_{m, 1} & b_{m, 2} & \ldots & b_{m, m-1} & b_{m, m} & c_{1, m} & c_{1, m-1} & \cdots & c_{12} & c_{11} \\ \hline c_{11} & c_{12} & \cdots & c_{1, m-1} & c_{1, m} & b_{m, m} & b_{m, m-1} & \cdots & b_{m, 2} & b_{m, 1} \\ c_{21} & c_{22} & \ldots & c_{2, m-1} & c_{2, m} & b_{m-1, m} & b_{m-1, m-1} & \cdots & b_{m-1,2} & b_{m-1,1} \\ \vdots & \vdots & . & \therefore & & & \ddots & \ddots & \vdots & \vdots \\ c_{m-1,1} & c_{m-1,2} & c_{m-1,3} & & & & & b_{23} & b_{22} & b_{21} \\ c_{m, 1} & c_{m, 2} & & & & & & & b_{12} & b_{11}\end{array}\right)$

Then, the block matrices of this matrix are

$$
\begin{gathered}
\boldsymbol{B}=\left(\begin{array}{ccccc}
b_{11} & b_{12} & & & \\
b_{21} & b_{22} & b_{23} & & \\
\vdots & \vdots & \ddots & \ddots & \\
b_{m-1,1} & b_{m-1,2} & \cdots & b_{m-1, m-1} & b_{m-1, m} \\
b_{m, 1} & b_{m, 2} & \cdots & b_{m, m-1} & b_{m, m}
\end{array}\right) \text { and } \\
\boldsymbol{J}_{\boldsymbol{m}} \boldsymbol{C}=\left(\begin{array}{ccccc}
c_{11} & c_{12} & & & \\
c_{21} & c_{22} & c_{23} & & \\
\vdots & \vdots & \ddots & \ddots & \\
c_{m-1,1} & c_{m-1,2} & \cdots & c_{m-1, m-1} & c_{m-1, m} \\
c_{m, 1} & c_{m, 2} & \cdots & c_{m, m-1} & c_{m, m}
\end{array}\right)
\end{gathered}
$$


where both of them are Hessenberg matrices. There is special form as block matrices on centrosymmetric matrix. This condition is useful on computing determinant of centrosymmetric matrix using determinant of lower Hessenberg matrix.

From Lemma 2, there is an orthogonal matrix $\boldsymbol{Q}=\frac{\sqrt{2}}{2}\left(\begin{array}{cc}\boldsymbol{I}_{\boldsymbol{m}} & \boldsymbol{I}_{\boldsymbol{m}} \\ -\boldsymbol{J}_{\boldsymbol{m}} & \boldsymbol{J}_{\boldsymbol{m}}\end{array}\right)$, then

$$
Q^{T} A Q=\left(\begin{array}{cc}
B-J_{m} C & 0_{m} \\
0_{m} & B+J_{m} C
\end{array}\right)=\left(\begin{array}{cc}
M & 0_{m} \\
0_{m} & N
\end{array}\right)
$$

where $\boldsymbol{M}=\boldsymbol{B}-\boldsymbol{J}_{\boldsymbol{m}} \boldsymbol{C}, \boldsymbol{N}=\boldsymbol{B}+\boldsymbol{J}_{\boldsymbol{m}} \boldsymbol{C}$ and $\boldsymbol{M}, \boldsymbol{N}$ are Hessenberg matrices.

By the same way [16] about the determinant of lower Hessenberg matrix, then we can assume

and

$$
\widetilde{\boldsymbol{M}}^{-1}=\left(\begin{array}{cc}
\boldsymbol{\alpha}_{M} & \boldsymbol{W}_{\boldsymbol{M}} \\
r_{M} & \boldsymbol{\beta}_{M}^{T}
\end{array}\right), \widetilde{\boldsymbol{N}}^{-1}=\left(\begin{array}{cc}
\boldsymbol{\alpha}_{\boldsymbol{N}} & \boldsymbol{W}_{\boldsymbol{N}} \\
r_{N} & \boldsymbol{\beta}_{N}^{T}
\end{array}\right)
$$

$$
\widetilde{\boldsymbol{M}}=\left(\begin{array}{cc}
\boldsymbol{e}_{1}^{T} & 0 \\
\boldsymbol{M} & \boldsymbol{e}_{\boldsymbol{m}}
\end{array}\right), \widetilde{\boldsymbol{N}}=\left(\begin{array}{cc}
\boldsymbol{e}_{1}^{T} & 0 \\
\boldsymbol{N} & \boldsymbol{e}_{\boldsymbol{m}}
\end{array}\right) \text {. }
$$

Therefore $r_{M}, r_{N} \neq 0$, if $r_{M}, r_{N}=0$ than $\boldsymbol{\alpha}_{\boldsymbol{M}}, \boldsymbol{\alpha}_{\boldsymbol{N}}=0$ since $\boldsymbol{M} \boldsymbol{\alpha}_{\boldsymbol{M}}=0$ and $\boldsymbol{N} \boldsymbol{\alpha}_{\boldsymbol{N}}=$ 0 . It implies that $\widetilde{\boldsymbol{M}}^{\mathbf{- 1}}$ and $\widetilde{\boldsymbol{N}}^{\mathbf{- 1}}$ are nonsingular matrix, which are contradiction.

c. Compute determinant of centrosymmetric matrix

The block of centrosymmetric matrix as explained before, it is formed as

$$
\boldsymbol{A}=\boldsymbol{Q}\left(\begin{array}{cc}
\boldsymbol{B}-\boldsymbol{J}_{m} \boldsymbol{C} & \mathbf{0}_{m} \\
\mathbf{0}_{m} & \boldsymbol{B}+\boldsymbol{J}_{m} \boldsymbol{C}
\end{array}\right) \boldsymbol{Q}^{T}=\boldsymbol{Q}\left(\begin{array}{cc}
\boldsymbol{M} & \mathbf{0}_{m} \\
\mathbf{0}_{m} & \boldsymbol{N}
\end{array}\right) \boldsymbol{Q}^{T}
$$

then

$$
\operatorname{det}(\boldsymbol{A})=\operatorname{det}(\boldsymbol{Q}) \cdot \operatorname{det}\left(\begin{array}{cc}
\boldsymbol{M} & \mathbf{0}_{\boldsymbol{m}} \\
\mathbf{0}_{\boldsymbol{m}} & \boldsymbol{N}
\end{array}\right) \cdot \operatorname{det}\left(\boldsymbol{Q}^{T}\right)=\operatorname{det}(\boldsymbol{M}) \cdot \operatorname{det}(\boldsymbol{N}) .
$$

The theorem of determinant of centrosymmetric matrix with lower Hessenberg as block matrices is obtained as follows.

Theorem $3[14,15]$. Let $A$ is centrosymmetric matrix and it's block matrices are $M, N$ as Hessenberg matrices which are described before, then

$$
\operatorname{det}(\boldsymbol{A})=r_{N} \cdot r_{M} \cdot \prod_{i=1}^{m-1}\left(g_{i, i+1} \cdot q_{i, i+1}\right) .
$$

This step is the algorithm determinant of centrosymmetric matrix with lower Hessenberg as block matrix. The other blocks matrices also appear on centrosymmetric matrix are upper Hessenberg, centrosymmetric, lower triangular, upper triangular, diagonal, tridiagonal matrix. Furthermore, some numerical examples of determinant of centrosymmetric matrix with different block matrices are given.

\subsection{Numerical Experiences}

This part will show the different block matrices arising at some examples of centrosymmetric matrices by applying the previous algorithm.

Example 1. [14,15] Given the following centrosymmetric matrix

$$
\boldsymbol{A}=\left(\begin{array}{llllllll}
1 & 1 & 0 & 0 & 0 & 0 & 2 & 1 \\
0 & 2 & 2 & 0 & 0 & 1 & 3 & 0 \\
1 & 2 & 2 & 1 & 2 & 2 & 2 & 1 \\
1 & 0 & 1 & 1 & 4 & 1 & 3 & 2 \\
2 & 3 & 1 & 4 & 1 & 1 & 0 & 1 \\
1 & 2 & 2 & 2 & 1 & 2 & 2 & 1 \\
0 & 3 & 1 & 0 & 0 & 2 & 2 & 0 \\
1 & 2 & 0 & 0 & 0 & 0 & 1 & 1
\end{array}\right) \text { where } \boldsymbol{B}=\left(\begin{array}{llll}
1 & 1 & 0 & 0 \\
0 & 2 & 2 & 0 \\
1 & 2 & 2 & 1 \\
1 & 0 & 1 & 1
\end{array}\right) \text { is lower Hessenberg as }
$$


block matrix, $\boldsymbol{C}=\left(\begin{array}{cccc}2 & 3 & 1 & 4 \\ 1 & 2 & 2 & 2 \\ 0 & 3 & 1 & 0 \\ 1 & 2 & 0 & 0\end{array}\right)$ and $\boldsymbol{J}_{4}=\left(\begin{array}{cccc}0 & 0 & 0 & 1 \\ 0 & 0 & 1 & 0 \\ 0 & 1 & 0 & 0 \\ 1 & 0 & 0 & 0\end{array}\right)$

Therefore, $\boldsymbol{M}=\boldsymbol{B}-\boldsymbol{J}_{\mathbf{4}} \boldsymbol{C}=\left(\begin{array}{cccc}0 & -1 & 0 & 0 \\ 0 & -1 & 1 & 0 \\ 0 & 0 & 0 & -1 \\ -1 & -3 & 0 & -3\end{array}\right), \boldsymbol{N}=\boldsymbol{B}+\boldsymbol{J}_{\mathbf{4}} \boldsymbol{C}=\left(\begin{array}{llll}2 & 3 & 0 & 0 \\ 0 & 5 & 3 & 0 \\ 2 & 4 & 4 & 3 \\ 3 & 3 & 2 & 5\end{array}\right)$ are lower Hessenberg matrices.

Based on the algorithm before, this step can be continued caused by both of block matrices are lower Hessenberg form.

Determinant of this matrix can be solved with $\widetilde{\boldsymbol{M}}=\left(\begin{array}{ccccc}1 & 0 & 0 & 0 & 0 \\ 0 & -1 & 0 & 0 & 0 \\ 0 & -1 & 1 & 0 & 0 \\ 0 & 0 & 0 & -1 & 0 \\ -1 & -3 & 0 & -3 & 1\end{array}\right)$,

$\widetilde{\boldsymbol{N}}=\left(\begin{array}{lllll}1 & 0 & 0 & 0 & 0 \\ 2 & 3 & 0 & 0 & 0 \\ 0 & 5 & 3 & 0 & 0 \\ 2 & 4 & 4 & 3 & 0 \\ 3 & 3 & 2 & 5 & 1\end{array}\right)$. So, it can be founded $\widetilde{\boldsymbol{M}}^{-1}=\left(\begin{array}{ccccc}1 & 0 & 0 & 0 & 0 \\ 0 & -1 & 0 & 0 & 0 \\ 0 & -1 & 1 & 0 & 0 \\ 0 & 0 & 0 & -1 & 0 \\ -1 & -3 & 0 & -3 & 1\end{array}\right)$ and

$\widetilde{N}^{-1}=\left(\begin{array}{ccccc}1 & 0 & 0 & 0 & 0 \\ -0.6667 & 0.3333 & 0 & 0 & 0 \\ 1.1111 & -0.5556 & 0.3333 & 0 & 0 \\ -1.2593 & 0.2963 & -0.4444 & 0.3333 & 0 \\ 3.0741 & -1.3704 & 1.5556 & -1.6667 & 1\end{array}\right)$.

Therefore,

$r_{M}=1, r_{N}=3.0741 \quad$ and $\quad \prod_{i=1}^{4}\left(g_{i, i+1}\right)=(-1)(1)(-1)=1, \quad \prod_{i=1}^{4}\left(q_{i, i+1}\right)=$ $(3)(3)(3)=27$

then

$$
\operatorname{det}(\boldsymbol{H})=r_{M} \cdot r_{N} \cdot \prod_{i=1}^{m-1}\left(g_{i, i+1} \cdot q_{i, i+1}\right)=(1)(3.0741)(1)(27)=83.0007 .
$$

Example 2. Consider the centrosymmetric matrix $\boldsymbol{A}=\left(\begin{array}{llllllll}2 & 3 & 1 & 4 & 1 & 1 & 0 & 1 \\ 1 & 2 & 2 & 2 & 1 & 2 & 2 & 1 \\ 0 & 3 & 1 & 0 & 0 & 2 & 2 & 0 \\ 1 & 2 & 0 & 0 & 0 & 0 & 1 & 1 \\ 1 & 1 & 0 & 0 & 0 & 0 & 2 & 1 \\ 0 & 2 & 2 & 0 & 0 & 1 & 3 & 0 \\ 1 & 2 & 2 & 1 & 2 & 2 & 2 & 1 \\ 1 & 0 & 1 & 1 & 4 & 1 & 3 & 2\end{array}\right)$

with $\boldsymbol{B}$ is not lower Hessenberg as block matrix. By Lemma 2, the matrix of $\boldsymbol{A}$ is formed as block matrices

$\boldsymbol{B}-\boldsymbol{J}_{4} \boldsymbol{C}=\boldsymbol{M}=\left(\begin{array}{cccc}1 & 3 & 0 & 3 \\ 0 & 0 & 0 & 1 \\ 0 & 1 & -1 & 0 \\ 0 & 1 & 0 & 0\end{array}\right), \boldsymbol{B}+\boldsymbol{J}_{4} \boldsymbol{C}=\boldsymbol{N}=\left(\begin{array}{cccc}3 & 3 & 2 & 5 \\ 2 & 4 & 4 & 3 \\ 0 & 5 & 3 & 0 \\ 2 & 3 & 0 & 0\end{array}\right)$ 
and we have

$$
\widetilde{\boldsymbol{M}}=\left(\begin{array}{ccccc}
1 & 0 & 0 & 0 & 0 \\
1 & 3 & 0 & 3 & 0 \\
0 & 0 & 0 & 1 & 0 \\
0 & 1 & -1 & 0 & 0 \\
0 & 1 & 0 & 0 & 1
\end{array}\right), \widetilde{\boldsymbol{N}}=\left(\begin{array}{ccccc}
1 & 0 & 0 & 0 & 0 \\
3 & 3 & 2 & 5 & 0 \\
2 & 4 & 4 & 3 & 0 \\
0 & 5 & 3 & 0 & 0 \\
2 & 3 & 0 & 0 & 1
\end{array}\right)
$$

which are not lower triangular matrices form, then the algorithm is not suitable for this condition. This algorithm is stopped because of non lower Hessenberg of its block matrices $\boldsymbol{M}$ and $\boldsymbol{N}$.

Example 3. Consider centrosymmetric matrix $\boldsymbol{A}=\left(\begin{array}{llllllll}1 & 1 & 0 & 0 & 4 & 1 & 3 & 2 \\ 0 & 2 & 2 & 0 & 2 & 2 & 2 & 1 \\ 1 & 2 & 2 & 1 & 0 & 1 & 3 & 0 \\ 1 & 0 & 1 & 1 & 0 & 0 & 2 & 1 \\ 1 & 2 & 0 & 0 & 1 & 1 & 0 & 1 \\ 0 & 3 & 1 & 0 & 1 & 2 & 2 & 1 \\ 1 & 2 & 2 & 2 & 0 & 2 & 2 & 0 \\ 2 & 3 & 1 & 4 & 0 & 0 & 1 & 1\end{array}\right)$ has $\boldsymbol{B}$ and $\boldsymbol{C}$ are lower Hessenberg as block matrices. Thus, we have

$$
\begin{aligned}
\boldsymbol{M} & =\left(\begin{array}{cccc}
-1 & -2 & -1 & -4 \\
-1 & 0 & 0 & -2 \\
1 & -1 & 1 & 1 \\
0 & -2 & 1 & 1
\end{array}\right), \boldsymbol{N}=\left(\begin{array}{llll}
3 & 4 & 1 & 4 \\
1 & 4 & 4 & 2 \\
1 & 5 & 3 & 1 \\
2 & 2 & 1 & 1
\end{array}\right) \text {, then we have } \\
\widetilde{\boldsymbol{M}} & =\left(\begin{array}{ccccc}
1 & 0 & 0 & 0 & 0 \\
-1 & -2 & -1 & -4 & 0 \\
-1 & 0 & 0 & -2 & 0 \\
1 & -1 & 1 & 1 & 0 \\
0 & -2 & 1 & 1 & 1
\end{array}\right), \widetilde{\boldsymbol{N}}=\left(\begin{array}{lllll}
1 & 0 & 0 & 0 & 0 \\
3 & 4 & 1 & 4 & 0 \\
1 & 4 & 4 & 2 & 0 \\
1 & 5 & 3 & 1 & 0 \\
2 & 2 & 1 & 1 & 1
\end{array}\right)
\end{aligned}
$$

which are not lower triangular matrices form, then the algorithm is not suitable for this condition caused by the lower Hessenberg matrices appear on two block matrices $\boldsymbol{B}, \boldsymbol{C}$.

Example 4. Given $\boldsymbol{A}=\left(\begin{array}{cccccccc}2.5 & 1.5 & 0 & 0 & 2.5 & 1.5 & 1 & 0 \\ 1.5 & 2.5 & 1.5 & 0 & 1.5 & 2.5 & 1.5 & 1 \\ 1 & 1.5 & 2.5 & 1.5 & 1 & 1.5 & 2.5 & 1.5 \\ 0 & 1 & 1.5 & 2.5 & 0 & 1 & 1.5 & 2.5 \\ 2.5 & 1.5 & 0 & 0 & 2.5 & 1.5 & 1 & 0 \\ 1.5 & 2.5 & 1.5 & 0 & 1.5 & 2.5 & 1.5 & 1 \\ 1 & 1.5 & 2.5 & 1.5 & 1 & 1.5 & 2.5 & 1.5 \\ 0 & 1 & 1.5 & 2.5 & 0 & 1 & 1.5 & 2.5\end{array}\right)$ then

$\boldsymbol{M}=\boldsymbol{B}-\boldsymbol{J}_{4} \boldsymbol{C}=\left(\begin{array}{cccc}2.5 & 0.5 & -0.5 & -2.5 \\ 0.5 & 1 & -1 & -0.5 \\ -0.5 & -1 & 1 & 0.5 \\ -2.5 & -0.5 & 0.5 & 2.5\end{array}\right)$

$\boldsymbol{N}=\boldsymbol{B}+\boldsymbol{J}_{\mathbf{4}} \boldsymbol{C}=\left(\begin{array}{cccc}2.5 & 2.5 & 2.5 & 2.5 \\ 2.5 & 4 & 4 & 2.5 \\ 2.5 & 4 & 4 & 2.5 \\ 2.5 & 2.5 & 2.5 & 2.5\end{array}\right)$ are centrosymmetric matrices too.

It can be seen that centrosymmetric matrix can appear on centrosymmetric matrix as block matrix. It seen that block matrices of matrix are $\boldsymbol{B}=\boldsymbol{C}$ cause be zero on determinant and the algorithm is not suitable for this condition. 
Example 5. Given $\boldsymbol{A}=\left(\begin{array}{llllllll}1 & 2 & 3 & 4 & 1 & 0 & 4 & 3 \\ 2 & 1 & 2 & 3 & 0 & 1 & 0 & 4 \\ 3 & 2 & 1 & 2 & 4 & 0 & 1 & 0 \\ 4 & 3 & 2 & 1 & 3 & 4 & 0 & 1 \\ 1 & 0 & 4 & 3 & 1 & 2 & 3 & 4 \\ 0 & 1 & 0 & 4 & 2 & 1 & 2 & 3 \\ 4 & 0 & 1 & 0 & 3 & 2 & 1 & 2 \\ 3 & 4 & 0 & 1 & 4 & 3 & 2 & 1\end{array}\right)$, then

$\boldsymbol{M}=\boldsymbol{B}-\boldsymbol{J}_{\mathbf{4}} \boldsymbol{C}=\left(\begin{array}{cccc}-2 & -2 & 3 & 3 \\ -2 & 1 & 1 & 3 \\ 3 & 1 & 1 & -2 \\ 3 & 3 & -2 & -2\end{array}\right), \quad \boldsymbol{N}=\boldsymbol{B}+\boldsymbol{J}_{\mathbf{4}} \boldsymbol{C}=\left(\begin{array}{cccc}4 & 6 & 3 & 5 \\ 6 & 1 & 3 & 3 \\ 3 & 3 & 1 & 6 \\ 5 & 3 & 6 & 4\end{array}\right) \quad$ are centrosymmetric matrices too.

It can be seen that centrosymmetric matrix can appear on centrosymmetric matrix as block matrix. It can be seen that block matrices of matrix are $\boldsymbol{B} \neq \boldsymbol{C}$ caused determinant is nonzero and the algorithm is not suitable for this condition. Moreover, there no lower Hessenberg form as block matrix at matrix $\boldsymbol{B}$.

Example 6. Consider the centrosymmetric matrix $\boldsymbol{A}=\left(\begin{array}{llllllll}1 & 0 & 0 & 0 & 3 & 1 & 3 & 2 \\ 2 & 3 & 0 & 0 & 0 & 3 & 2 & 2 \\ 3 & 2 & 1 & 0 & 0 & 0 & 2 & 1 \\ 2 & 3 & 1 & 2 & 0 & 0 & 0 & 2 \\ 2 & 0 & 0 & 0 & 2 & 1 & 3 & 2 \\ 1 & 2 & 0 & 0 & 0 & 1 & 2 & 3 \\ 2 & 2 & 3 & 0 & 0 & 0 & 3 & 2 \\ 2 & 3 & 1 & 3 & 0 & 0 & 0 & 1\end{array}\right)$ has tridiagonal matrix $\boldsymbol{B}$ and $\boldsymbol{C}$ as block matrices. By Lemma 2, the matrix of $\boldsymbol{A}$ is formed as block matrices

$\boldsymbol{B}-\boldsymbol{J}_{4} \boldsymbol{C}=\boldsymbol{M}=\left(\begin{array}{cccc}-1 & -3 & -1 & -3 \\ 0 & 1 & -3 & 0 \\ 2 & 0 & 1 & 0 \\ 0 & 3 & 1 & 2\end{array}\right), \boldsymbol{B}+\boldsymbol{J}_{4} \boldsymbol{C}=\boldsymbol{N}=\left(\begin{array}{cccc}3 & 3 & 1 & 3 \\ 4 & 5 & 3 & 0 \\ 4 & 4 & 1 & 0 \\ 4 & 3 & 1 & 2\end{array}\right)$

and we have

$\widetilde{\boldsymbol{M}}=\left(\begin{array}{ccccc}1 & 0 & 0 & 0 & 0 \\ -1 & -3 & -1 & -3 & 0 \\ 0 & 1 & -3 & 0 & 0 \\ 2 & 0 & 1 & 0 & 0 \\ 0 & 3 & 1 & 2 & 1\end{array}\right), \widetilde{\boldsymbol{N}}=\left(\begin{array}{lllll}1 & 0 & 0 & 0 & 0 \\ 3 & 3 & 1 & 3 & 0 \\ 4 & 5 & 3 & 0 & 0 \\ 4 & 4 & 1 & 0 & 0 \\ 4 & 3 & 1 & 2 & 1\end{array}\right)$

which are not lower triangular matrices form, then the algorithm is not suitable for this condition. The algorithm cannot be continued caused the block matrix is not lower Hessenberg form.

Example 7. Consider the centrosymmetric matrix

$\boldsymbol{A}=\left(\begin{array}{llllllll}1 & 2 & 3 & 2 & 3 & 0 & 0 & 0 \\ 0 & 3 & 2 & 3 & 1 & 3 & 0 & 0 \\ 0 & 0 & 1 & 1 & 3 & 2 & 2 & 0 \\ 0 & 0 & 0 & 2 & 2 & 2 & 1 & 2 \\ 2 & 1 & 2 & 2 & 2 & 0 & 0 & 0 \\ 0 & 2 & 2 & 3 & 1 & 1 & 0 & 0 \\ 0 & 0 & 3 & 1 & 3 & 2 & 3 & 0 \\ 0 & 0 & 0 & 3 & 2 & 3 & 2 & 1\end{array}\right)$ where block matrices $\boldsymbol{B}$ and $\boldsymbol{C}$ are upper Hessenberg 
form. By Lemma 2, the matrix of $\boldsymbol{A}$ is formed as block matrices

$$
\boldsymbol{B}-\boldsymbol{J}_{4} \boldsymbol{C}=\boldsymbol{M}=\left(\begin{array}{cccc}
1 & 2 & 3 & -1 \\
0 & 3 & -1 & 2 \\
0 & -2 & -1 & -2 \\
-2 & -1 & -2 & 0
\end{array}\right), \boldsymbol{B}+\boldsymbol{J}_{4} \boldsymbol{C}=\boldsymbol{N}=\left(\begin{array}{cccc}
1 & 2 & 3 & 5 \\
0 & 3 & 5 & 4 \\
0 & 2 & 3 & 4 \\
2 & 1 & 2 & 4
\end{array}\right)
$$

and we have

$$
\widetilde{\boldsymbol{M}}=\left(\begin{array}{ccccc}
1 & 0 & 0 & 0 & 0 \\
1 & 2 & 3 & -1 & 0 \\
0 & 3 & -1 & 2 & 0 \\
0 & -2 & -1 & -2 & 0 \\
-2 & -1 & -2 & 0 & 1
\end{array}\right), \widetilde{\boldsymbol{N}}=\left(\begin{array}{ccccc}
1 & 0 & 0 & 0 & 0 \\
1 & 2 & 3 & 5 & 0 \\
0 & 3 & 5 & 4 & 0 \\
0 & 2 & 3 & 4 & 0 \\
2 & 1 & 2 & 4 & 1
\end{array}\right) .
$$

which are not lower triangular matrices form, then the algorithm is not suitable for this condition. It happen caused by block matrix $\boldsymbol{B}$ is not lower Hessenberg matrix.

Example 8. Consider the centrosymmetric matrix

$\boldsymbol{A}=\left(\begin{array}{llllllll}1 & 0 & 0 & 0 & 8 & 0 & 0 & 0 \\ 0 & 2 & 0 & 0 & 0 & 7 & 0 & 0 \\ 0 & 0 & 3 & 0 & 0 & 0 & 6 & 0 \\ 0 & 0 & 0 & 4 & 0 & 0 & 0 & 5 \\ 5 & 0 & 0 & 0 & 4 & 0 & 0 & 0 \\ 0 & 6 & 0 & 0 & 0 & 3 & 0 & 0 \\ 0 & 0 & 7 & 0 & 0 & 0 & 2 & 0 \\ 0 & 0 & 0 & 8 & 0 & 0 & 0 & 1\end{array}\right)$ where $\boldsymbol{B}$ and $\boldsymbol{C}$ are diagonal matrices as block matrix.

By Lemma 2, the matrix of $\boldsymbol{A}$ is formed as block matrices

$\boldsymbol{B}-\boldsymbol{J}_{4} \boldsymbol{C}=\boldsymbol{M}=\left(\begin{array}{cccc}1 & 0 & 0 & -8 \\ 0 & 2 & -7 & 0 \\ 0 & -6 & 3 & 0 \\ -5 & 0 & 0 & 4\end{array}\right), \boldsymbol{B}+\boldsymbol{J}_{4} \boldsymbol{C}=\boldsymbol{N}=\left(\begin{array}{cccc}1 & 0 & 0 & 8 \\ 0 & 2 & 7 & 0 \\ 0 & 6 & 3 & 0 \\ 5 & 0 & 0 & 4\end{array}\right)$

and we have

$$
\widetilde{\boldsymbol{M}}=\left(\begin{array}{ccccc}
1 & 0 & 0 & 0 & 0 \\
1 & 0 & 0 & -8 & 0 \\
0 & 2 & -7 & 0 & 0 \\
0 & -6 & 3 & 0 & 0 \\
-5 & 0 & 0 & 4 & 1
\end{array}\right), \widetilde{\boldsymbol{N}}=\left(\begin{array}{ccccc}
1 & 0 & 0 & 0 & 0 \\
1 & 0 & 0 & 8 & 0 \\
0 & 2 & 7 & 0 & 0 \\
0 & 6 & 3 & 0 & 0 \\
5 & 0 & 0 & 4 & 1
\end{array}\right) .
$$

which are not lower triangular matrices form, then the algorithm is not suitable for this condition. It cannot be continued because of lower Hessenberg form does not appear as block matrix.

Example 9. Consider the centrosymmetric matrix $\boldsymbol{A}=\left(\begin{array}{llllllll}2 & 1 & 0 & 0 & 4 & 3 & 0 & 0 \\ 1 & 2 & 1 & 0 & 3 & 4 & 3 & 0 \\ 0 & 1 & 2 & 1 & 0 & 3 & 4 & 3 \\ 0 & 0 & 1 & 2 & 0 & 0 & 3 & 4 \\ 4 & 3 & 0 & 0 & 2 & 1 & 0 & 0 \\ 3 & 4 & 3 & 0 & 1 & 2 & 1 & 0 \\ 0 & 3 & 4 & 3 & 0 & 1 & 2 & 1 \\ 0 & 0 & 3 & 4 & 0 & 0 & 1 & 2\end{array}\right)$

has $\boldsymbol{B}$ and $\boldsymbol{C}$ are tridiagonal matrix as block matrices. By Lemma 2, the matrix of $\boldsymbol{A}$ is formed as block matrices 
$\boldsymbol{B}-\boldsymbol{J}_{4} \boldsymbol{C}=\boldsymbol{M}=\left(\begin{array}{cccc}2 & 1 & -3 & -4 \\ 1 & -1 & -3 & -3 \\ -3 & -3 & -1 & 1 \\ -4 & -3 & 1 & 2\end{array}\right), \boldsymbol{B}+\boldsymbol{J}_{4} \boldsymbol{C}=\boldsymbol{N}=\left(\begin{array}{cccc}2 & 1 & 3 & 4 \\ 1 & 5 & 5 & 3 \\ 3 & 5 & 5 & 1 \\ 4 & 3 & 1 & 2\end{array}\right)$

and we have

$$
\widetilde{\boldsymbol{M}}=\left(\begin{array}{ccccc}
1 & 0 & 0 & 0 & 0 \\
2 & 1 & -3 & -4 & 0 \\
1 & -1 & -3 & -3 & 0 \\
-3 & -3 & -1 & 1 & 0 \\
-4 & -3 & 1 & 2 & 1
\end{array}\right), \widetilde{\boldsymbol{N}}=\left(\begin{array}{lllll}
1 & 0 & 0 & 0 & 0 \\
2 & 1 & 3 & 4 & 0 \\
1 & 5 & 5 & 3 & 0 \\
3 & 5 & 5 & 1 & 0 \\
4 & 3 & 1 & 2 & 1
\end{array}\right)
$$

which are not lower triangular matrices form, then the algorithm is not suitable for this condition.

\section{Conclusions}

The algorithm of determinant centrosymmetric matrix is presented efficiently. This algorithm focusing with the centrosymmetric matrix with lower Hessenberg matrix as block matrix. But there are some block matrices appear as block of centrosymmetric matrices such as upper Hessenberg, centrosymmetric, lower triangular, upper triangular, diagonal, tridiagonal matrix. Therefore, this algorithm is only on compute the determinant of centrosymmetric matrix with lower Hessenberg as block matrix. For the other block matrices, this algorithm does not work well.

\section{References}

[1] Zhong-Yun Liu, "Some properties of centrosymmetric matrices", Appl. Math. Comput. 141 pp. 297-306, 2003.

[2] Hongyi Li, Di Zhao, Fei Dai and Donglin Su, "On the spectral radius of a nonnegative centrosymmetric matrix", Appl. Math. Comput., 218 (9) pp. 4962-4966, 2012.

[3] Melman, "Symmetric centrosymmetric matrix-vector multiplication", Linear Algebra and Its Appl., 320 pp.193-198, 2000.

[4] Iyad T. Abu-Jeib, "Centrosymmetric matrices : properties and an alternative approach", Canadian Applied Mathematics Quarterly, 10 pp. 429-445, 2000.

[5] Alan L. Andrew, "Eigenvector of certain matrices", Linear Algebra and Its Appl., 7 pp. 151-162, 2000.

[6] Charles F Van Loan and Joseph P Vokt, "Approximating matrices with multiple symmetries", SIAM J.Matrix Anal.Appl., 36(3) pp.974-993, 2015.

[7] Dattatreya A.V. Rao and K. Venkata Ramana, "On LU decomposition of a centrosymmetric matrix", Information Sciences, 63 pp. 3-10, 2000.

[8] Konrad Burnik, "A structure-preserving QR factorization for centrosymmetric real matrices", Linear Algebra and Its Applications, 484 pp. 356-378, 2015.

[9] Gene H. Golub and Charles F. Van Loan, "Matrix Computations, third ed.", Johns Hopkins University Press, Baltimore and London, (1996).

[10] Tomohiro Sogabe, "On a two-term recurrence for the determinant of a general matrix", Appl. Math. Comput., 187 pp. 785-788, 2007.

[11] Mohamed Elouafi and A.D. Aiat Hadj, "A new recursive algorithm for inverting Hessenberg matrices", Appl. Math. Comput., 214 pp. 497-499, 2009.

[12] F Bunger, "Inverse, determinant, eigenvalues, and eigenvectors of real symmetric Toeplitz matrices with linearly increasing entries", Linear Algebra and its 
Applications.459 pp.595-619, 2014.

[13] Mohamed Elouafi and A.D. Aiat Hadj, "A new recursive algorithm for inverting Hessenberg matrices", Appl. Math. Comput., 214 pp. 497-499, 2009.

[14] Di Zhao and Hongyi Li, "On the computation of inverse and determinant of a kind of special matrices", Appl. Math. Comput., 250 pp. 721-726, 2015.

[15] N Khasanah, Farikhin and B Surarso, "The algorithm of determinant of centrosymmetric matrix based on lower Hessenberg form", IOP Conf. Series : Journal of Physics : Conf. Series $824,2017$.

[16] Datta and Morgera, "On the reducibility of centrosymmetric matrices-Aplication in engineering problems", Circuits System Signal Process., 8 (1) pp. 71-95, 1989. 